\title{
POSITRON ANNIHILATION IN SOLID AND LIQUID $\mathrm{Bi}_{40} \mathrm{Sn}_{60}$ ALLOY
}

\author{
St. ChabiK ${ }^{a}$, W. Rudzińska ${ }^{a}$, M. Szuszkiewicz ${ }^{b}$ AND Cz. Szymański ${ }^{a}$ \\ ${ }^{a}$ Institute of Physics, Opole University, Oleska 48, 45-052 Opole, Poland \\ ${ }^{b}$ Institute of Experimental Physics, Wroclaw University \\ Pl. Maxa Borna 9, 50-204 Wrocław, Poland
}

The peak counting rate, $F(T)$, for $\mathrm{Bi}_{40} \mathrm{Sn}_{60}$ alloy in the temperature range from room temperature to $400^{\circ} \mathrm{C}$ was measured in three consecutive heating-cooling runs. Significant changes in $F(T)$ were observed in the temperature interval between the solidus and liquidus temperature. The magnitude of these anomalies systematically diminishes in consecutive heating-cooling runs. Noticeable changes of values of $F(T)$ parameter in the liquid alloy were observed, too. These effects were connected with trapping of positrons at the grain boundaries-defects and in the short-range ordered regions in the liquid alloy. Also microscopic structure of sample before and after measurements cycles was observed.

PACS numbers: 64.70.Dv, 61.72.Cc, 78.70.Bj

\section{Introduction}

The study on the nature of materials near the melting point of solid phase and near the crystallisation point of a liquid phase remains to be a matter of concern in both the fundamental research and practical applications. The melting process is particularly complex for eutectic alloys. Below the solidus temperature different types of crystal structures can coexist in these alloys. However, in the temperature slightly above the liquidus temperature the structure of metallic alloys can be represented as a mixed structure consisting of a simple liquid and the strongly correlated regions. like the solid state. The structures in solid and liquid phases are usually strongly defected. In the solid phase, vacancy-like defects and grain boundaries are present and in the liquid phase, inhomogeneity on th. nundaries between the structureless and short-range ordered regions can exist. the positron annihilation method is a very powerful technique for observing small inhomogeneous regions acting as positron trapping centers. In our previous paper [1] the results for $\mathrm{Bi}_{66.7} \mathrm{Sn}_{33.3}$ alloy were presented. Hysteresis in the peak coincidence counting rate dependence in the heating and cooling run was found. In this work we present results of similar measurements for $\mathrm{Bi}_{60} \mathrm{Sn}_{40}$ alloy. 


\section{Experimental}

The sample was prepared by melting together the granules of the $4 \mathrm{~N}$ pure tin and bismuth in the rectangular measuring vessel, while on the other side rectangular titanium foil ( $8 \mu \mathrm{m}$ thick) was mounted. This way positrons from ${ }^{22} \mathrm{Na}$ source penetrated the interior of the sample through titanium foil. After melting the sample was stored at $400^{\circ} \mathrm{C}$ and then slowly cooled to room temperature. The peak coincidence counting rate, $F(T)$, in three consecutive heating-cooling runs was measured. During the measurements the sample was kept in a protective argon atmosphere under atmospheric pressure. In addition, after the measuring runs microscopic structure of the sample was compared with the microstructure of sample formed in the same way by applying microscope Neophot 2. Finally, the crystal structure of the sample was examined by X-ray diffractometry.

\section{Results and discussion}

In Fig. 1 the $F(T)$ dependence for $\mathrm{Bi}_{40} \mathrm{Sn}_{60}$ alloy, measured in the temperature range between room temperature and $400^{\circ} \mathrm{C}$ in three consecutive heating and cooling runs is shown. The $F(T)$ parameter changes significantly mainly be-

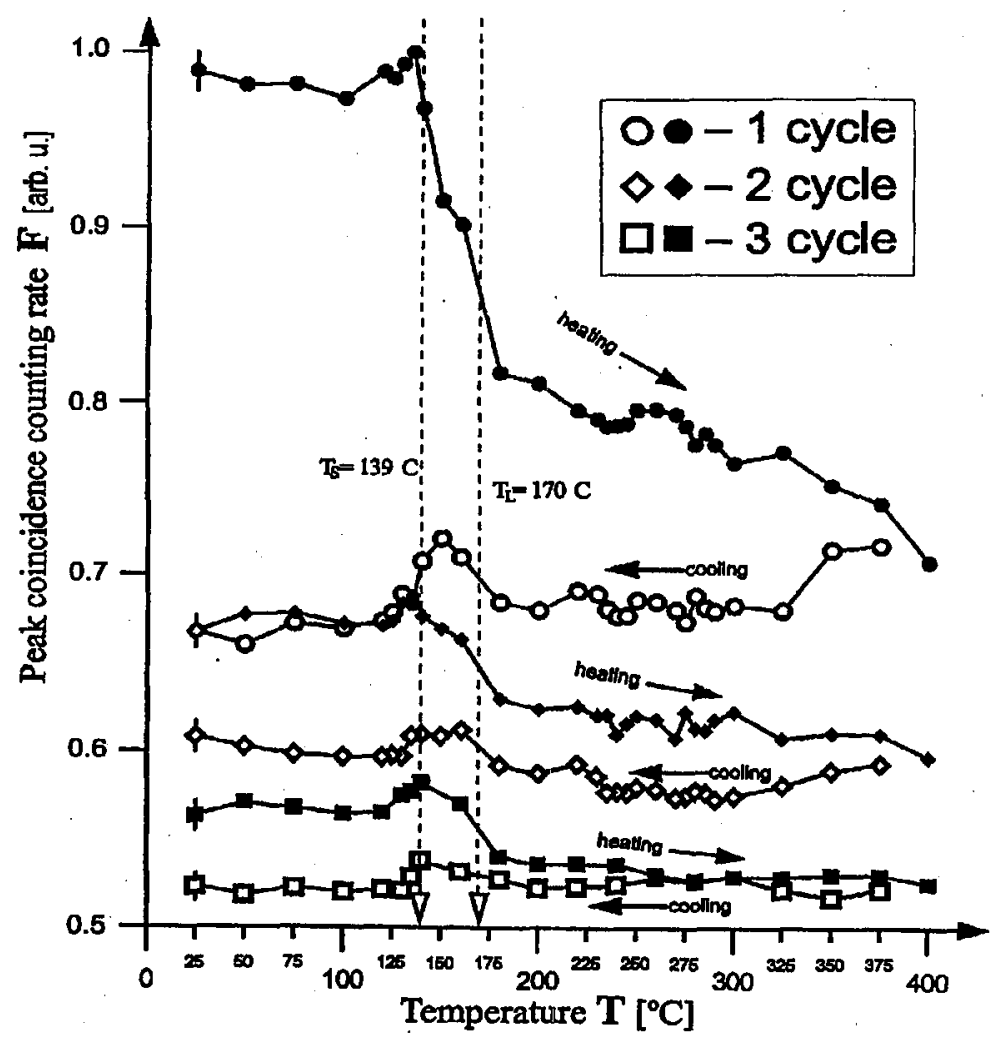

Fig. 1. Dependence of the peak coincidence counting rate for $\mathrm{Bi}_{40} \mathrm{Sn}_{60}$ alloy in the three consecutive heating-cooling runs. 
tween the solidus temperature $\left(T_{\mathrm{S}}\right)$ and liquidus temperature $\left(T_{\mathrm{L}}\right)$. Values of these changes decrease in the consecutive series of measurements. It is known that the structure of solid tin-bismuth eutectic alloy consists of separate Bi and Sn grains forming the so-called "Chinese-script" texture. The existence of pure tin and bismuth grains in the investigated $\mathrm{Bi}_{40} \mathrm{Sn}_{60}$ sample was also confirmed by X-ray measurements. The size of grains and contribution of the grain boundaries in the volume of alloy decreases after each measurement series. This effect causes the hysteresis in the peak counting rate measurements.

Hysteresis of the melting and solidification processes appears not only in positron annihilation measurements. Similar effect was observed in the ultrasonic pulse transmission measurements [2]. Also inhomogeneity of the structure of liquid alloy was found. The $F(T)$ dependence reflects rather the changes of defect state than the changes of alloy structure. It is known that the vacancy-like defects in solid bismuth do not trap positrons [3] and in solid tin the trapping of positrons at the same defects is very weak [4]. Thus $F(T)$ parameter between room temperature and $125^{\circ} \mathrm{C}$ in the each cycle is almost constant. Only above the temperature $125^{\circ} \mathrm{C}$ up to $T_{\mathrm{S}}$ the values of $F(T)$ parameter increase. This last effect may be attributed to the trapping of positrons at the $\mathrm{Sn}$ vacancies. The threshold temperature for this effect amounts about $130^{\circ} \mathrm{C}$. The possibility that this effect is caused by melting of sub-microcrystals of the alloys at temperature significantly lower than $T_{\mathrm{S}}$, as suggested in [3], seems to be less probable. As is shown in Fig. 1, distinct changes in the $F(T)$ dependence appears between $T_{\mathrm{S}}$ and $T_{\mathrm{L}}$, and in all heating-cooling
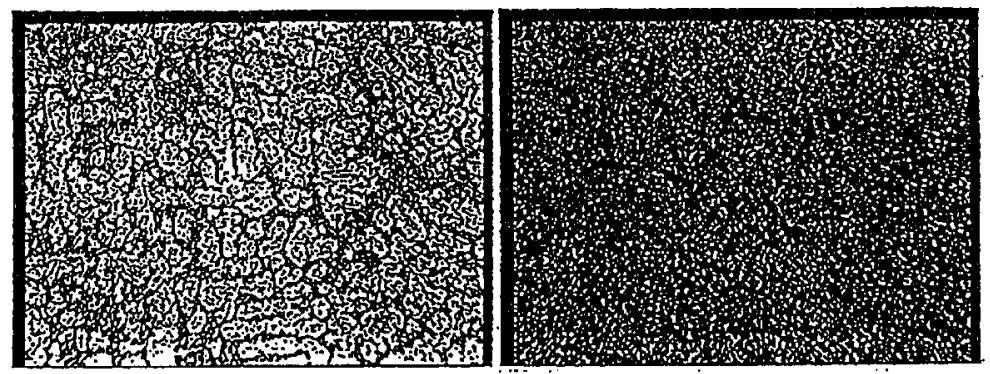

$\times 100$

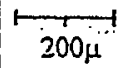

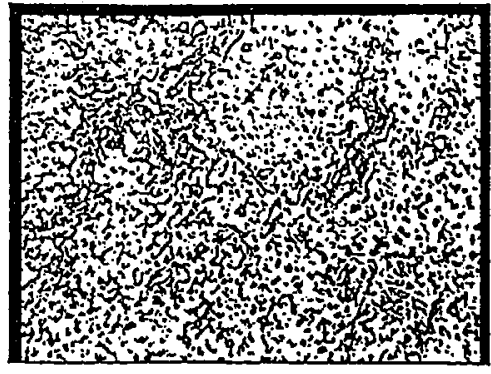

(a)

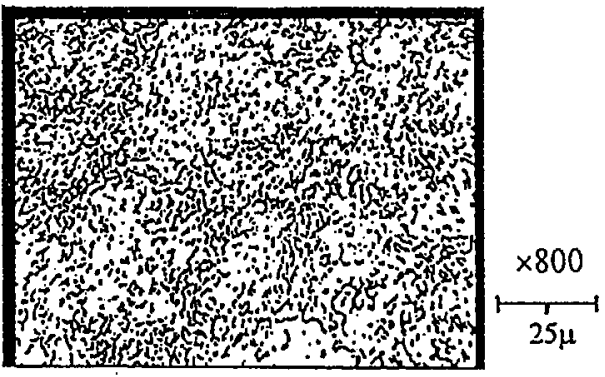

(b)

Fig. 2. Microstructure of $\mathrm{Bi}_{40} \mathrm{Sn}_{60}$ samples: (a) before and (b) after measurement. 
runs the values of $F(T)$ parameter are higher in the solid phase than those for the liquid. It can be assigned with positrons trapping at the grain boundaries, i.e. with defects in the solid alloy and with disintegration of these defects during the melting process.

Strong heterogeneity of the structure in mixed phases and liquid phase during the first and second heating runs becomes evident. In both cases the drop of the values of $F(T)$ parameter between $T_{\mathrm{S}}$ and $T_{\mathrm{L}}$ is very high and in the temperatures corresponding to melting point of $\mathrm{Sn}\left(232^{\circ} \mathrm{C}\right)$ and $\mathrm{Bi}\left(271^{\circ} \mathrm{C}\right)$ the changes of $F(T)$ are noticeable. Thus, the existence of short-range regions in the liquid alloy containing $\mathrm{Sn}$ and $\mathrm{Bi}$ atoms may be deduced. Similar effect for $\mathrm{Sn}_{66.7} \mathrm{Bi}_{33.3}$ alloy was previously observed [1]. Effect of homogeneity of the sample is visible in Fig. 2, where microstructures of the surface of sample before and after the measurement are shown.

\section{Conclusions}

The results of the present study demonstrate once more that the thermal history of sample strongly affects the homogeneity of its structure. Positron annihilation measurements can be used to detect grain boundaries defects and the inhomogeneities of liquid alloys structure. It results from the fact that the trapping of positrons is very effective at those defects. On the other hand, this phenomenon can be an interpretation of positron annihilation results (i.e. for recovery processes) for metals and alloys, particularly for eutectic alloys.

\section{References}

[1] St. Chabik, W. Rudzińska, M. Szuszkiewicz, Cz. Szymański, Nukleonika 42, 57 (1997).

[2] H. Kamioka, J. Phys. Soc. Jpn. 53, 1349 (1984).

[3] D. Segers, M. Dorikens, L. Dorikens-Vanpraet, Phys. Status Solidi A 48, 133 (1978).

[4] Nareshandra Shah, A.L. Catz, Phys. Rev. B 30, 2498 (1984). 